\title{
Fatigue in multiple sclerosis: Associations with clinical, MRI and CSF parameters
}

\author{
Viola Biberacher, Paul Schmidt, Rebecca C Selter, Verena Pernpeinter, Markus C Kowarik, \\ Benjamin Knier, Dorothea Buck, Muna-Miriam Hoshi, Thomas Korn, Achim Berthele, \\ Jan S Kirschke, Claus Zimmer, Bernhard Hemmer and Mark Mühlau
}

\section{Abstract}

Background: Damage of different brain structures has been related to fatigue. Alternatively, functional alterations of central nervous system (CNS) cells by the inflammatory milieu within the CNS may be responsible for the development of fatigue.

Aim: To investigate the effect of structural brain damage and inflammatory cerebrospinal fluid (CSF) changes on fatigue in multiple sclerosis (MS).

Methods: We determined the association of different clinical, CSF and magnetic resonance imaging (MRI) parameters with prevalence and severity of fatigue, as measured by the Fatigue Scale for Motor and Cognitive Functions in 68 early MS patients (discovery cohort). We validated our findings in two MS cohorts: the MRI validation cohort $(N=233)$ for the clinical and MRI parameters, and the CSF validation cohort $(N=81)$ for the clinical and CSF parameters.

Results: Fatigue was associated with clinical disability. Fatigue did not correlate with any CSF parameter but correlated negatively with total and cortical grey matter volume. However, when controlling for Expanded Disability Status Scale (EDSS) in a multivariate model, these associations lost significance.

Conclusion: Disability and disease duration best explain fatigue severity but none of the tested MRI or CSF parameter was reliably associated with fatigue.

Keywords: Multiple sclerosis, demyelinating diseases, fatigue, magnetic resonance imaging, flow cytometry, cerebrospinal fluid

Date received: 22 December 2016; revised: 24 April 2017; accepted: 28 April 2017

\section{Introduction}

Fatigue is one of the most prevalent symptoms in multiple sclerosis (MS) patients. It is present early in the disease course, ${ }^{1,2}$ can even precede other MS symptoms ${ }^{3}$ and predicts disease progression. ${ }^{1,4}$

The pathophysiology of fatigue is still unclear. Damage of specific brain structures and strategic localization of MS lesions have been related to fatigue in MS patients. However, results from previous studies are conflicting. ${ }^{5}$ Alternatively, the inflammatory milieu in the central nervous system (CNS) compartment of MS patients might produce symptoms of fatigue by inducing functional alterations of CNS intrinsic cells. This hypothesis is supported by the fact that other autoimmune diseases, chronic or acute infections and malignancies are characterized by fatigue similar to MS. ${ }^{5}$ Studies investigating immunologic markers for fatigue in the blood of MS patients failed to identify a meaningful parameter. ${ }^{5}$ Inflammatory mechanisms may mainly occur in the CNS compartment and might not be measurable in blood samples of MS patients. ${ }^{5}$

In the study at hand, we tested possible associations of fatigue with the morphometric imaging parameters suggested until now ${ }^{5}$ and immunologic markers in a cohort of 68 early MS patients. In a second step, we controlled for clinical disability and depression which are both associated with fatigue. ${ }^{6}$

Immunologic markers were investigated in the cerebrospinal fluid (CSF) compartment, which might reflect inflammatory changes in the CNS parenchyma
Multiple Sclerosis Journal

2018, Vol. 24(8) 1115-1125

DOI: $10.1177 /$

1352458517712078

(C) The Author(s), 2017. Reprints and permissions: http://www.sagepub.co.uk/ journalsPermissions.nav
Correspondence to:

V Biberacher

Department of Neurology, Klinikum rechts der Isar,

Technische Universität

München, Ismaninger Str. 22, D-81675 Munich, Germany. viola.biberacher@tum.de

Viola Biberacher

Mark Mühlau

Department of Neurology,

Klinikum rechts der Isar,

Technische Universität

München, Munich, Germany/

Neuroimaging Center,

Technische Universität

München, Munich, Germany

Paul Schmidt

Department of Neurology,

Klinikum rechts der Isar,

Technische Universität

München, Munich, Germany/

Neuroimaging Center,

Technische Universität

München, Munich, Germany/

Department of Statistics,

Ludwig-Maximilians-

Universität München,

Munich, Germany

Rebecca C Selter

Verena Pernpeinter

Benjamin Knier

Dorothea Buck

Muna-Miriam Hoshi

Achim Berthele

Department of Neurology,

Klinikum rechts der Isar,

Technische Universität

München, Munich, Germany

Markus C Kowarik

Thomas Korn

Bernhard Hemmer

Department of Neurology,

Klinikum rechts der Isar,

Technische Universität

München, Munich, Germany/

Munich Cluster for Systems

Neurology (SyNergy),

Ludwig-Maximilians-

Universität München,

Munich, Germany

Jan S Kirschke

Claus Zimmer

Department of

Neuroradiology, Klinikum

rechts der Isar, Technische

Universität München,

Munich, Germany 


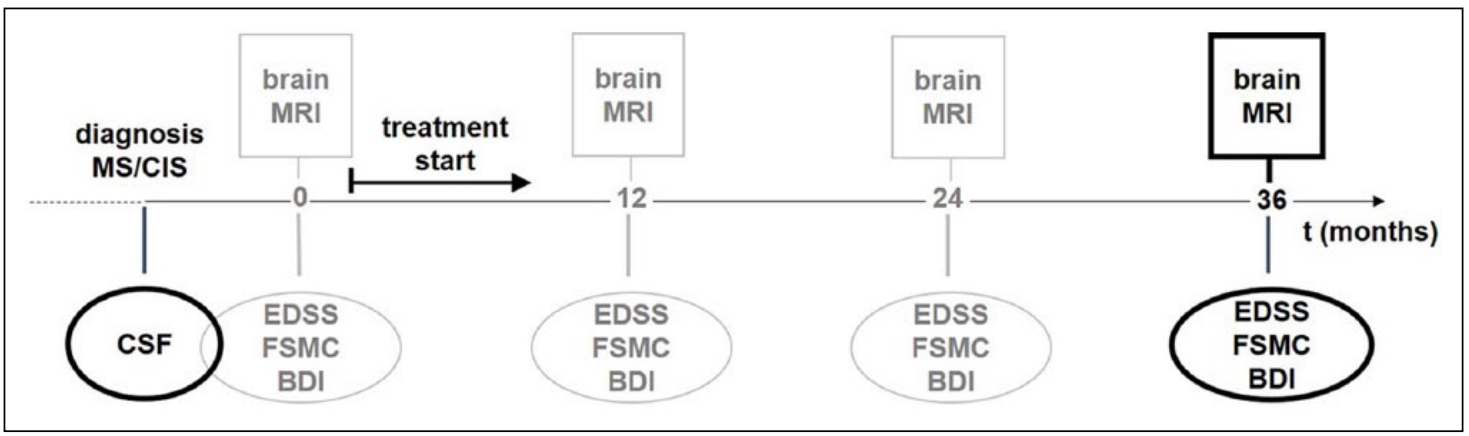

Figure 1. Study design.

A schematic overview of the study design is given. CSF is withdrawn at disease onset before the start of an immunomodulatory treatment. Afterwards, standardized brain MRI scans, clinical and neuropsychological tests are performed annually. Focusing on 3-year follow-up, three cohorts were investigated: discovery cohort, patients with CSF analysis at disease onset, clinical and MRI follow-up data; CSF validation cohort, patients with CSF analysis at disease onset and clinical follow-up data; and MRI validation cohort, patients with brain MRI and simultaneous clinical tests.

BDI: Becks Depression Inventory II; CIS: clinically isolated syndrome; CSF: cerebrospinal fluid; EDSS: Expanded Disability Status Scale; FSMC: Fatigue Scale for Motor and Cognitive Functions; MRI: magnetic resonance imaging; $t$ : time.

to a certain degree. We were particularly interested in the distribution of CSF immune cells, as immunologic dysregulation ${ }^{7}$ with alterations in B- ${ }^{8}$ and T- 9,10 cell function had also been described in patients with chronic fatigue syndrome (CFS). B-cell depleting therapies have been shown to ameliorate fatigue in $\mathrm{CFS}^{11}$ and autoimmune diseases ${ }^{12}$ but did not influence fatigue in a trial with $75 \mathrm{MS}$ patients. ${ }^{13} \mathrm{We}$ aimed to validate our findings in two separate cohorts of early MS patients, the magnetic resonance imaging (MRI) and CSF validation cohort.

\section{Materials and methods}

\section{Subjects and study design}

This study was performed in accordance with the Code of Ethics of the World Medical Association (Declaration of Helsinki) for experiments involving humans and was approved by the local ethics committee. We retrospectively analysed data that were collected in an observational study (TUM-MS) since January 2009 at the Department of Neurology at the Technical University of Munich. The study design is as follows (see also Figure 1): All patients with a diagnosis of MS according to current McDonald criteria ${ }^{14}$ or clinically isolated syndrome (CIS) that are regularly followed in our MS outpatient clinic were asked for their informed consent to participate in the TUM-MS study and to provide their data and biomaterials for scientific purposes. CIS was defined as first demyelinating event suspicious of MS accompanied by at least two brain white matter (WM) lesions detected by MRI. If disease diagnosis was made in our clinic, CSF was drawn as part of routine clinical work-up. After study inclusion, standardized brain
MRI scans, clinical and neuropsychological tests were performed annually within 30 days. Disability was quantified by Expanded Disability Status Scale (EDSS) by the treating neurologist in the MS outpatient unit; fatigue was assessed by Fatigue Scale for Motor and Cognitive Functions (FSMC). FSMC is a 20-item questionnaire developed to differentiate grade motor and cognitive fatigue in MS patients. ${ }^{15}$ Depression was evaluated by Beck Depression Inventory-II (BDI). ${ }^{16}$

For the study at hand, we excluded patients with relevant comorbidities as malignancies, autoimmune and psychiatric diseases. Patients with depression were included, as depression affects around $25 \%$ of MS patients with a lifetime prevalence of up to $54 \% .{ }^{17}$ Excluding these patients might introduce a systematic bias. Nevertheless, one patient with long-standing major depression before the onset of MS symptoms was excluded. We also excluded patients with a relapse or EDSS $>4$ at time of fatigue assessment. For CSF analysis, only therapy-naïve patients were selected (no immunomodulatory drugs, no corticosteroids within the past 30 days). Afterwards, they received different immunomodulatory drugs according to the recommendation of their treating physician. We focused on fatigue scores 3 years after disease diagnosis and investigated three cohorts of MS patients: The discovery cohort consisted of 68 patients of whom all investigated items were available (CSF at disease onset, follow-up brain MRI and clinical tests). The MRI validation cohort consisted of 233 patients of whom brain MRI scans with simultaneous clinical and neuropsychological tests were available. The CSF validation cohort consisted of 81 patients of whom CSF at disease onset and follow-up clinical tests were available. 
Table 1. Basic data and clinical examination.

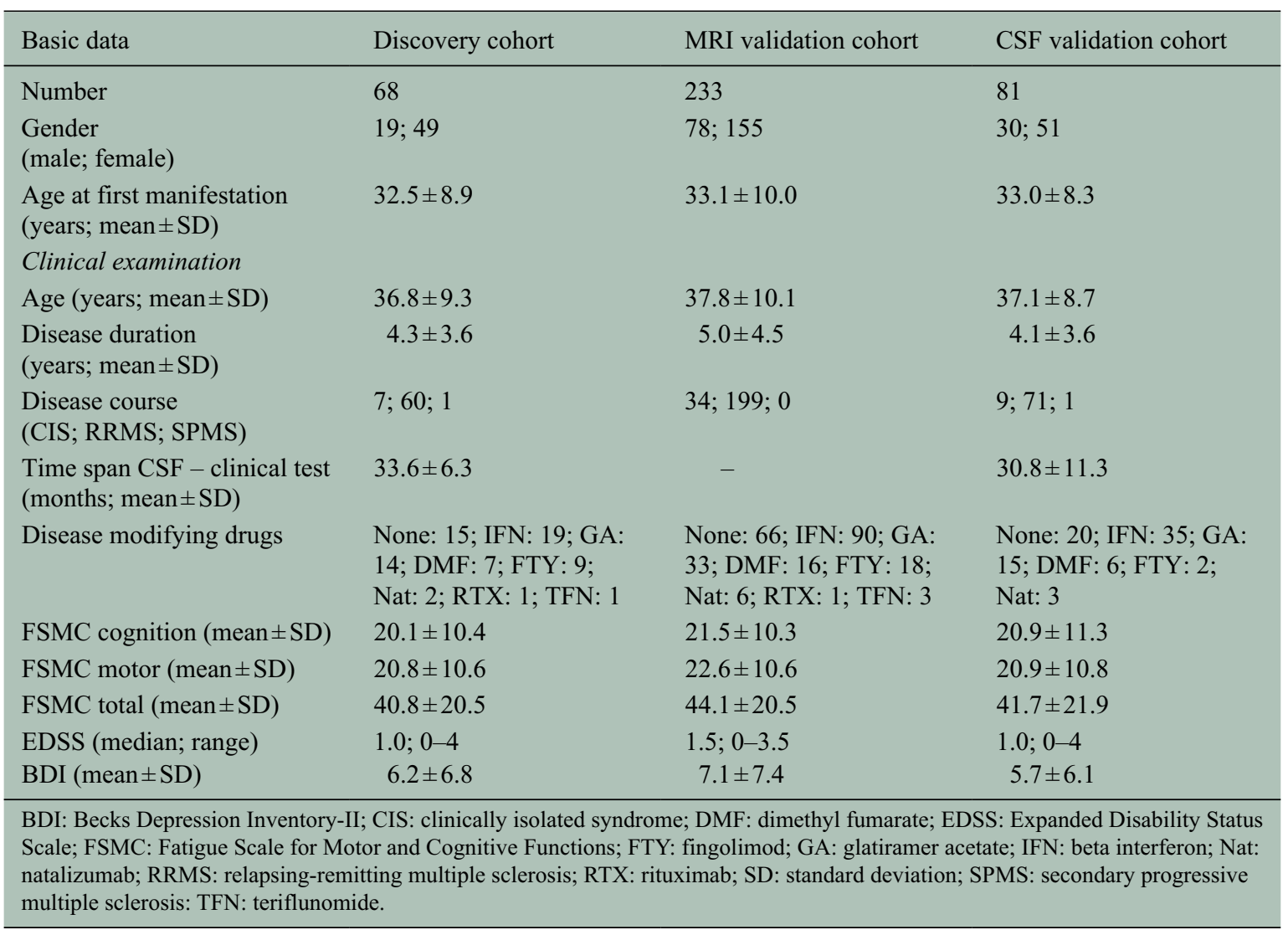

Patient characteristics, clinical and neuropsychological tests at follow-up are summarized in Table 1.

\section{Analysis of CSF}

\section{Routine CSF parameters}

CSF was drawn as part of routine clinical work-up. Peripheral blood was taken immediately after lumbar puncture. CSF cells were counted manually in a Fuchs Rosenthal chamber. CSF glucose and lactate levels were measured by an enzymatic-amperometric method using chip-sensor technology (EKF Biosen C-Line). Albumin $\mathrm{CSF} /$ serum ratios and intrathecal immunoglobulin synthesis were determined by nephelometry (Siemens ProSpec ${ }^{\circledR}$ ). Fluorescence-associated cell sorting (FACS) of CSF immune cells was performed as described previously. ${ }^{18}$ The following antibodies were used for staining: cluster of differentiation (CD) 45, V450; CD3, APC-Cy7; CD4, PerCP (all BD Bioscience); CD8, PE-Cy7; CD14, FITC; CD19, ECD; CD138, PE; and CD56, APC (all Beckman Coulter). We stained $\mathrm{CD} 4+\mathrm{T}$ cells $(\mathrm{CD} 45+\mathrm{CD} 3+\mathrm{CD} 4+)$, $\mathrm{CD} 8+\mathrm{T}$ cells $(\mathrm{CD} 45+\mathrm{CD} 3+\mathrm{CD} 8+)$, monocytes
(CD45+CD14+), natural killer (NK) cells (CD45+CD56+), B cells (CD45+CD19+CD138-) and plasmablasts $(\mathrm{CD} 45+\mathrm{CD} 19+\mathrm{CD} 138+)$. The percentage of each subpopulation was determined in relation to all $\mathrm{CD} 45$ positive single cells using FlowJo (version 10.1). In the discovery cohort, B cells and plasmablasts staining was missing in seven patients and monocytes staining was missing in two patients. CSF parameters are summarized in Table 2.

\section{MRI}

\section{Scanning protocol}

All brain images were acquired on the same 3T scanner (Achieva, Philips, The Netherlands). The scanning protocol included 3D GRE T1-weighted (w) sequence (orientation: 170 contiguous sagittal 1-mm slices; field of view: $240 \times 240 \mathrm{~mm}^{2}$; voxel size: $1.0 \times 1.0 \times 1.0 \mathrm{~mm}^{3}$; repetition time (TR): $9 \mathrm{~ms}$; echo time (TE): $4 \mathrm{~ms}$ ), and 3D FLAIR sequence (orientation: 144 contiguous axial $1.5-\mathrm{mm}$ slices; field of view: $230 \times 185 \mathrm{~mm}^{2}$; voxel size: $1.0 \times 1.0 \times 1.5 \mathrm{~mm}^{3}$; TR: $10^{4} \mathrm{~ms}$; TE: $140 \mathrm{~ms}$; TI: $2750 \mathrm{~ms}$ ). 
Table 2. CSF analysis.

\begin{tabular}{|c|c|c|}
\hline CSF analysis & Discovery cohort & CSF validation cohort \\
\hline Number & 68 & 81 \\
\hline $\begin{array}{l}\text { Relapse within } 30 \text { days before spinal tap } \\
\text { (yes; no; unknown) }\end{array}$ & $54 ; 14 ; 0$ & $67 ; 11 ; 3$ \\
\hline $\begin{array}{l}\text { Cell count } \\
\text { count } / \mu \mathrm{L} ; \text { mean } \pm \mathrm{SD} \\
\text { Norm: }<3 / \mu \mathrm{L}\end{array}$ & $10.4 \pm 16.9$ & $12.3 \pm 17.7$ \\
\hline $\begin{array}{l}\text { Glucose } \\
(\mathrm{mg} / \mathrm{dL} ; \text { mean } \pm \mathrm{SD}) \\
\text { Norm: } 30-80 \mathrm{mg} / \mathrm{dL}\end{array}$ & $59.0 \pm 8.3$ & $61.4 \pm 9.4$ \\
\hline $\begin{array}{l}\text { Lactate } \\
(\mathrm{mmol} / \mathrm{L} \text { mean } \pm \mathrm{SD}) \\
\text { Norm: }<2.5 \mathrm{mmol} / \mathrm{L}\end{array}$ & $1.6 \pm 0.3$ & $1.6 \pm 0.3$ \\
\hline $\begin{array}{l}\text { Albumin ratio } 10^{-3} \\
(\text { mean } \pm \mathrm{SD}) \\
\text { Norm: }<6.5\end{array}$ & $6.5 \pm 3.2$ & $6.1 \pm 2.4$ \\
\hline $\begin{array}{l}\text { Intrathecal IgG synthesis } \\
\text { (no; yes; mean } \pm \text { SD) }\end{array}$ & $32 ; 36 ; 35.2 \pm 21.4$ & $42 ; 39 ; 35.7 \pm 19.4$ \\
\hline $\begin{array}{l}\text { Intrathecal IgA synthesis } \\
\text { (no; yes; mean } \pm \text { SD) }\end{array}$ & $61 ; 7 ; 25.3 \pm 25.9$ & $78 ; 3 ; 36.7 \pm 29.8$ \\
\hline $\begin{array}{l}\text { Intrathecal IgM synthesis } \\
\text { (no; yes; mean } \pm \text { SD) }\end{array}$ & $61 ; 7 ; 34.9 \pm 16.9$ & $67 ; 14 ; 33.1 \pm 21.0$ \\
\hline $\begin{array}{l}\text { Oligoclonal bands } \\
\text { (no; intermediate; yes) }\end{array}$ & $7 ; 12 ; 49$ & $6 ; 9 ; 66$ \\
\hline $\begin{array}{l}\text { CD4 }+ \text { T cells } \\
(\% \text { of CD } 45+\text { cells; mean } \pm \mathrm{SD})\end{array}$ & $66.7 \pm 8.2$ & $65.4 \pm 9.6$ \\
\hline $\begin{array}{l}\text { CD } 8+T \text { cells } \\
(\% \text { of CD } 45+\text { cells; mean } \pm \text { SD })\end{array}$ & $18.3 \pm 5.5$ & $17.5 \pm 6.2$ \\
\hline $\begin{array}{l}\text { CD4+/CD8 ratio } \\
(\text { mean } \pm \text { SD })\end{array}$ & $4.1 \pm 1.7$ & $4.3 \pm 2.0$ \\
\hline $\begin{array}{l}\text { CD } 19+\text { B cells } \\
(\% \text { of CD } 45+\text { cells; mean } \pm \text { SD })\end{array}$ & $3.0 \pm 2.8(N=62)$ & $2.8 \pm 1.9$ \\
\hline $\begin{array}{l}\text { CD } 19+\mathrm{CD} 138+\text { plasmablasts } \\
(\% \text { of CD } 45+\text { cells; mean } \pm \mathrm{SD})\end{array}$ & $0.6 \pm 0.6(N=62)$ & $0.6 \pm 0.6$ \\
\hline $\begin{array}{l}\text { CD56+ natural killer cells } \\
(\% \text { of CD } 45+\text { cells; mean } \pm \text { SD })\end{array}$ & $2.7 \pm 1.4$ & $2.1 \pm 1.1$ \\
\hline $\begin{array}{l}\text { CD14+ monocytes } \\
(\% \text { of CD } 45+\text { cells; mean } \pm \text { SD })\end{array}$ & $2.5 \pm 3.6(N=66)$ & $2.0 \pm 5.2$ \\
\hline
\end{tabular}

\section{T2-hyperintense WM lesions}

T2-hyperintense WM lesions were segmented from FLAIR and T1-w images by a lesion growth algorithm as implemented in the lesion segmentation tool (LST) toolbox version 2.0.15 (www.statistical-modelling.de/lst.html) for SPM12 (http://www.fil.ion.ucl. ac.uk/spm). Lesion maps were normalized before extraction of lesion volumes. Brainstem and cerebellar WM lesion volumes were determined by masking with brainstem and cerebellar WM masks from LONI Probabilistic Brain Atlas (LPBA40). ${ }^{19}$ Lesions were filled with normal appearing WM intensities in T1-w images by $\mathrm{LST}^{20} \mathrm{~A}$ natural logarithmic transformation was applied to all lesion volumes to approximate normal distribution.

\section{Segmentation of brain compartments}

Brain compartments were segmented with the computational anatomy toolbox (CAT12, version 916, $\mathrm{http}: / / \mathrm{dbm} . n e u r o . u n i-j e n a . d e / c a t /)$ as implemented in SPM12. We used SPM12 version 6685 for our analysis. We run the segmentation pipeline with the default settings that proceeds as follows: Lesion-filled T1-w images are normalized to Montreal Neurological Institute (MNI) template, segmented into the tissue 
classes of grey matter (GM) and WM, and corrected for signal inhomogeneities (correction of bias-field). Segmented images are scaled with the amount of volume changes resulting from normalization (modulation).$^{21}$ Before extraction of brain compartments, all voxels, classified as lesions by LST, were set to 0 in the GM image and to 1 in the WM image in order to correct for possible lesion filling errors.

Regional volumes were extracted from GM and WM images with the following atlases: LPBA $40^{19}$ for putamen, caudate nucleus, cerebellar cortex, brainstem and cerebellar WM; neuromorphometrics atlas (http://neuromorphometrics.com/) for thalamus, hippocampus and accumbens nucleus; and $\mathrm{CoBrA}^{22}$ for amygdala. For cerebral cortex, we used an own mask. $^{23}$ Total intracranial volume (TIV) was estimated by a reverse brain mask method. ${ }^{24}$ All MRI parameters are summarized in Table 3.

\section{Voxel-wise analysis}

In case of significant associations of global brain volumes with fatigue, we performed voxel-wise correlation in SPM12. Therefore, normalized GM images were smoothed with an 8-mm Gaussian kernel. Lesion maps are normalized by applying the deformation matrix derived from the corresponding lesion-filled T1-w image and smoothed with a 12-mm Gaussian kernel. Analysis of GM images was corrected for age and, in a second step, for EDSS. We used a False Discovery Rate of $<0.05$ to account for multiple testing.

\section{Quality assurance and plausibility control}

Quality of standard CSF parameters was assured by regular interlaboratory tests. For plausibility control of FACS data, we confirmed the correlation of CSF plasmablasts count with intrathecal IgG synthesis ${ }^{18}$ in the discovery $(r=0.432 ; p<0.0001)$ and the CSF validation cohort $(r=0.515 ; p=0.001)$. MRI data were checked by correlating age, ${ }^{25} \mathrm{EDSS}^{26}$ and WM lesion volume ${ }^{27}$ with different GM volumes (Figure 2).

\section{Statistical analysis}

We used IBM SPSS Statistics version 23 and R-environment for statistical computing ${ }^{28}$ to analyse and plot data. We investigated the effect of demographic and clinical parameters on fatigue severity by Pearson's correlation analysis (age, disease duration, EDSS and BDI), unpaired $t$-test (gender) and analysis of variance (ANOVA; disease modifying drugs; categorical variable for each drug). We performed partial correlation analyses with FSMC and the extracted brain MRI parameters, correcting for age, gender and total intracranial volume. We performed partial correlation analyses with all CSF parameters, correcting for age and gender. To consider multicollinearity and to correct for multiple testing, a joint analysis of the effect of all CSF and MRI variables on FSMC was performed by elastic net regularized regression approach. This approach uses a mixture of L1 (Lasso regression) and L2 penalty (Ridge regression), thus it is able to perform shrinkage and variable selection simultaneously. For estimation, we used the EBglmnet package (https:// CRAN.R-project.org $/$ package $=$ EBglmnet $)^{28}$ which implements an empirical Bayes approach. Optimal mixture parameters for the penalties were estimated using a leave-one-out cross validation.

\section{Results}

Prevalence of fatigue and association with clinical parameters

Motor and cognitive fatigue were highly correlated (all $R^{2} \geqslant 0.82 ; p<0.0001$ ). In the following, we will report the results for the FSMC sum score only.

FSMC correlated significantly with disability (EDSS) in all three cohorts (discovery, MRI and CSF validation cohorts; $r=0.54,0.46$ and 0.35 , respectively; all $p \mathrm{~s}<0.0001)$. All correlations remained significant when age and gender were included as confounders.

The distribution of BDI scores and the association with fatigue are illustrated in Figure 3. All patients with moderate or severe depression also had symptoms of fatigue, but not vice versa. Fatigue correlated significantly with BDI scores (discovery, MRI and CSF validation cohorts, $r=0.77,0.66$ and 0.73 , respectively; all $p \mathrm{~s}<0.0001)$. We decided to repeat some of the following correlation analyses after exclusion of patients with moderate and severe depression to ensure that these outliers were not primarily responsible for the observed correlation.

In the MRI validation cohort, fatigue increased significantly with disease duration $(r=0.17, p=0.008)$. The discovery cohort and the CSF validation cohort were not suitable for correlation of fatigue with disease duration as the clinical examination was performed at a standardized interval after diagnosis.

The immunomodulatory therapy did not have a significant effect on fatigue in any cohort (all $p \mathrm{~s}>0.2$ ). 
Table 3. Morphometric brain volume measures.

\begin{tabular}{|c|c|c|}
\hline MRI data & Discovery cohort & MRI validation cohort \\
\hline Number & 68 & 233 \\
\hline $\begin{array}{l}\text { Total intracranial volume } \\
(\mathrm{mL} ; \text { mean } \pm \mathrm{SD})\end{array}$ & $1091.6 \pm 99.7$ & $1368.8 \pm 128.0$ \\
\hline $\begin{array}{l}\text { Grey matter } \\
(\mathrm{mL} ; \text { mean } \pm \mathrm{SD})\end{array}$ & $601.7 \pm 50.2$ & $609.0 \pm 58.3$ \\
\hline $\begin{array}{l}\text { Cerebral cortex } \\
(\mathrm{mL} ; \text { mean } \pm \mathrm{SD})\end{array}$ & $555.5 \pm 47.0$ & $562.4 \pm 54.2$ \\
\hline $\begin{array}{l}\text { Putamen } \\
(\mathrm{mL} ; \text { mean } \pm \mathrm{SD})\end{array}$ & $8.5 \pm 1.0$ & $8.6 \pm 1.1$ \\
\hline $\begin{array}{l}\text { Caudate nucleus } \\
(\mathrm{mL} ; \text { mean } \pm \mathrm{SD})\end{array}$ & $6.5 \pm 0.8$ & $6.5 \pm 0.9$ \\
\hline $\begin{array}{l}\text { Thalamus } \\
(\mathrm{mL} ; \text { mean } \pm \mathrm{SD})\end{array}$ & $9.8 \pm 1.3$ & $9.7 \pm 1.6$ \\
\hline $\begin{array}{l}\text { Hippocampus } \\
(\mathrm{mL} ; \text { mean } \pm \mathrm{SD})\end{array}$ & $6.2 \pm 0.6$ & $6.2 \pm 0.6$ \\
\hline $\begin{array}{l}\text { Amygdala } \\
(\mathrm{mL} ; \text { mean } \pm \mathrm{SD})\end{array}$ & $3.0 \pm 0.3$ & $3.0 \pm 0.3$ \\
\hline $\begin{array}{l}\text { Accumbens nucleus } \\
(\mathrm{mL} ; \text { mean } \pm \mathrm{SD})\end{array}$ & $0.8 \pm 0.1$ & $0.8 \pm 0.1$ \\
\hline $\begin{array}{l}\text { Cerebellar cortex } \\
(\mathrm{mL} ; \text { mean } \pm \mathrm{SD})\end{array}$ & $85.4 \pm 7.5$ & $86.0 \pm 8.5$ \\
\hline $\begin{array}{l}\text { White matter } \\
(\mathrm{mL} ; \text { mean } \pm \mathrm{SD})\end{array}$ & $497.6 \pm 55.0$ & $503.7 \pm 52.8$ \\
\hline $\begin{array}{l}\text { Supratentorial white matter } \\
(\mathrm{mL} ; \text { mean } \pm \mathrm{SD})\end{array}$ & $429.6 \pm 49.4$ & $435.8 \pm 47.4$ \\
\hline $\begin{array}{l}\text { Brainstem white matter } \\
(\mathrm{mL} ; \text { mean } \pm \mathrm{SD})\end{array}$ & $27.7 \pm 2.9$ & $27.7 \pm 2.8$ \\
\hline $\begin{array}{l}\text { Cerebellar white matter } \\
(\mathrm{mL} ; \text { mean } \pm \mathrm{SD})\end{array}$ & $40.3 \pm 4.6$ & $40.2 \pm 4.7$ \\
\hline $\begin{array}{l}\text { White matter lesions } \\
(\mathrm{mL} ; \text { mean } \pm \mathrm{SD})\end{array}$ & $6.5 \pm 9.7$ & $6.7 \pm 10.6$ \\
\hline $\begin{array}{l}\text { Supratentorial white matter lesions } \\
(\mathrm{mL} ; \text { mean } \pm \mathrm{SD})\end{array}$ & $6.5 \pm 9.7$ & $6.7 \pm 10.6$ \\
\hline $\begin{array}{l}\text { Brainstem white matter lesions } \\
(\mathrm{mL} ; \text { mean } \pm \mathrm{SD})\end{array}$ & $0.01 \pm 0.03$ & $0.01 \pm 0.07$ \\
\hline $\begin{array}{l}\text { Cerebellar white matter lesions } \\
(\mathrm{mL} ; \text { mean } \pm \mathrm{SD})\end{array}$ & $0.01 \pm 0.04$ & $0.01 \pm 0.04$ \\
\hline
\end{tabular}

\section{MRI measures and fatigue}

Correlation analysis of fatigue with all extracted MRI parameters did not indicate any significant correlation in the discovery cohort. As significant correlations have been described in other studies, ${ }^{5}$ we decided to repeat the analysis in the MRI validation cohort in order to increase statistical power. We found significant negative correlations of motor fatigue with volume of total $(r=-0.139, p=0.036)$ and cortical $(r=-0.141, p=0.033)$ GM. Exclusion of patients with moderate or severe depression did not change these results (GM: $r=-0.166$, $p=0.016$; cortical GM: $r=-0.164, p=0.017)$. None of these correlations remained significant after additional correction for EDSS or disease duration (Figure 4). Likewise, in the multivariate model, fatigue was significantly associated with EDSS and BDI only, both in the discovery and the MRI validation cohort (all $p \mathbf{s}<0.01$ ).

Voxel-wise analysis of the MRI validation cohort confirmed this finding: We identified GM clusters that were significantly associated with fatigue in right insular cortex, left and right occipital pole, left inferior parietal lobule, left inferior temporal gyrus, left precentral gyrus, right middle frontal gyrus and right precuneus 


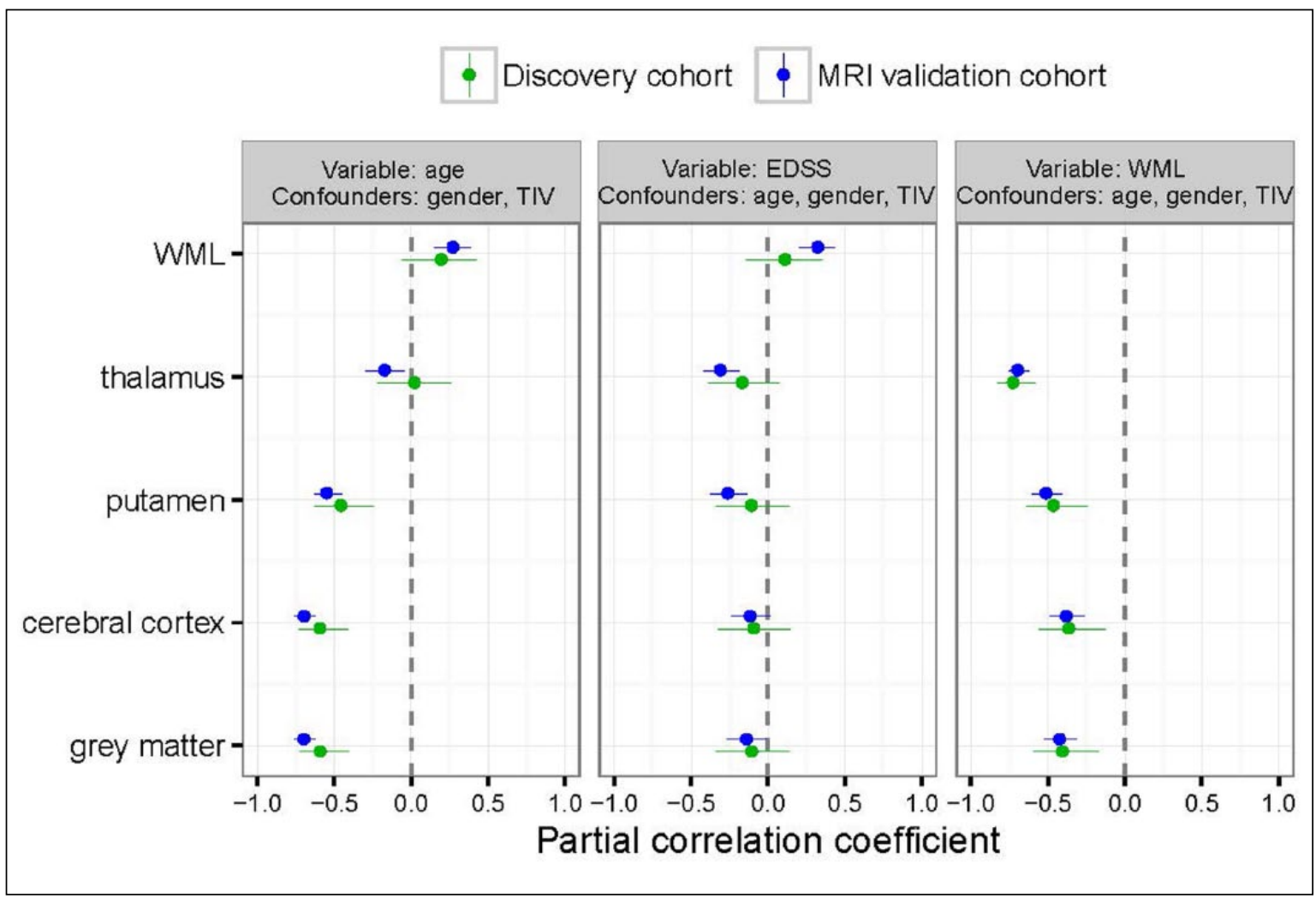

Figure 2. Plausibility control of MRI data by partial correlations.

Plausibility control of MRI data for the discovery cohort (green) and the MRI validation cohort (blue). Partial correlation coefficients and $95 \%$ confidence intervals are shown. Age was correlated with total white matter lesion volume and different grey matter structures, controlling for gender and total intracranial volume (left panel). EDSS (middle) and white matter lesion volume (right panel) were correlated with total white matter lesion volume and different grey matter structures, controlling for age, gender and total intracranial volume.

EDSS: Expanded Disability Status Scale; MRI: magnetic resonance imaging; TIV: total intracranial volume; WML: T2-hyperintense white matter lesion volume.

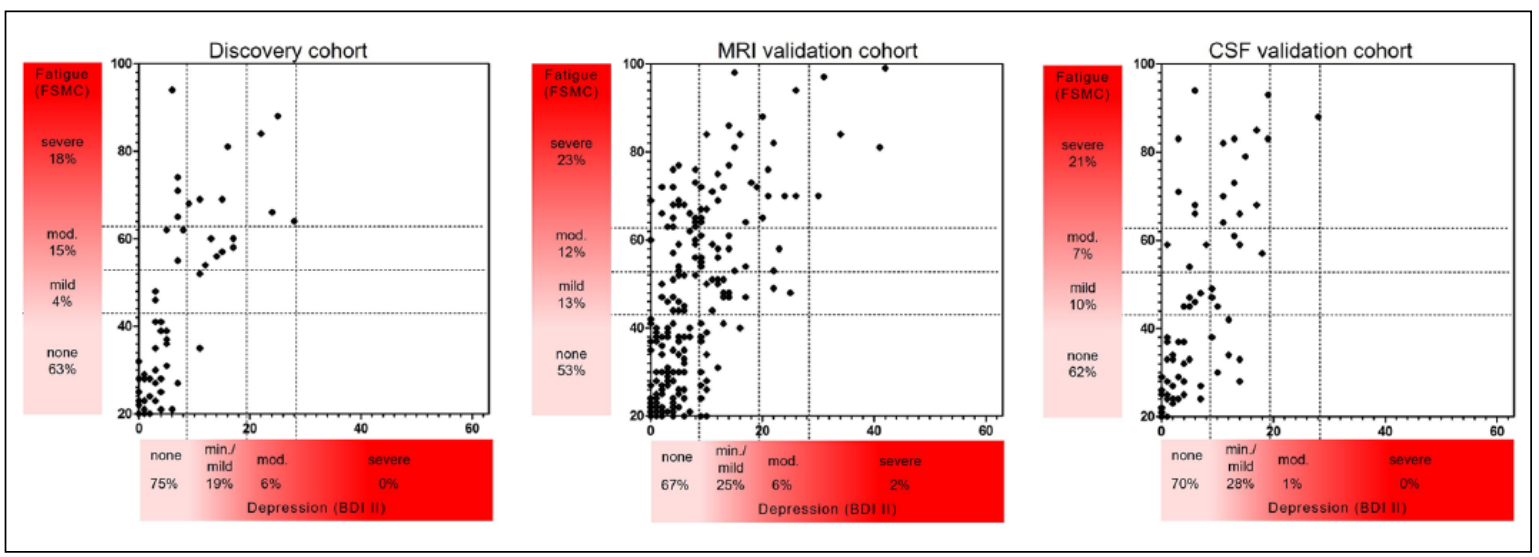

Figure 3. Prevalence of fatigue and depression.

The relationship of fatigue (Fatigue Scale for Motor and Cognitive Functions) with depression (Becks Depression Inventory-II) is shown for the discovery cohort (left panel), the MRI validation cohort (middle panel) and the CSF validation cohort (right panel).

BDI: Becks Depression Inventory-II; FSMC: Fatigue Scale for Motor and Cognitive Functions; min.: minimal; mod.: moderate.

cortex (all at a false discovery rate of $<0.05$ ), but none of them remained significant after inclusion of EDSS in the model. Voxel-wise analysis of lesion maps with fatigue did not show any significant cluster. 


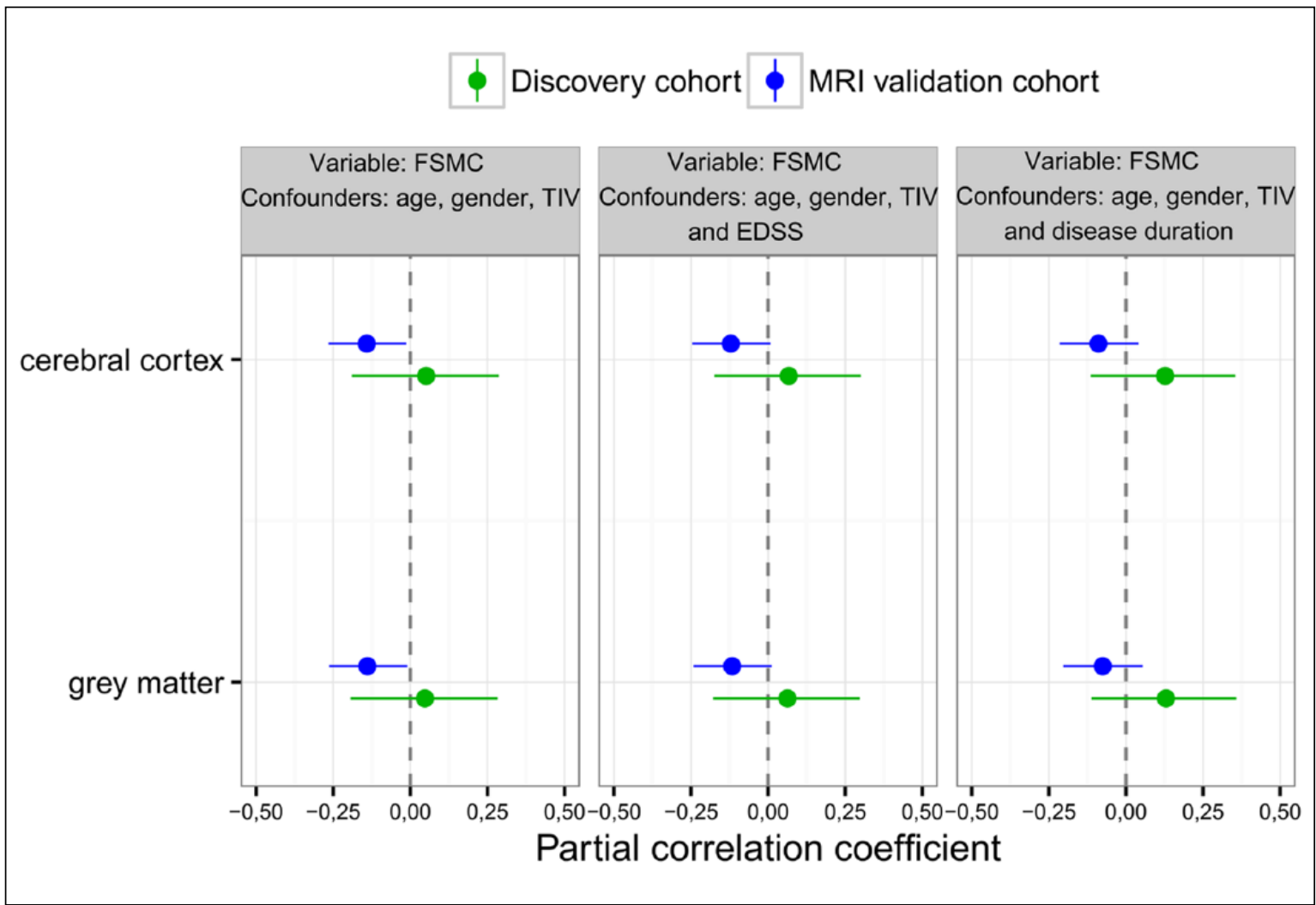

Figure 4. Partial correlation analyses of MRI parameters with fatigue.

Partial correlation coefficients and $95 \%$ confidence intervals of fatigue (Fatigue Scale for Motor and Cognitive Functions) with MRI parameters are shown for the discovery cohort (green) and the MRI validation cohort (blue). Only MRI parameters with a significant correlation in any analysis are shown. Confounders were age, gender and total intracranial volume (left panel); age, gender, total intracranial volume and Expanded Disability Status Scale (middle panel); and age, gender, total intracranial volume and disease duration (right panel).

EDSS: Expanded Disability Status Scale; FSMC: Fatigue Scale for Motor and Cognitive Functions; TIV: total intracranial volume.

\section{CSF measures and fatigue}

In the discovery cohort, none of the humoral CSF parameters was associated with fatigue. CSF CD4/ CD8 ratio showed a trend towards negative correlation with fatigue $(r=-0.234, p=0.059)$, which was not influenced by additional correction for relapse at time point of spinal tap (yes/no) and exclusion of patients with moderate and severe depression $(r=-0.235, p=0.068)$. However, this finding could not be replicated in the CSF validation cohort. Here, CSF CD4/CD8 ratio showed a trend towards positive correlation with fatigue $(r=0.192, p=0.09)$. In the multivariate model, none of the CSF parameters correlated with fatigue, neither in the discovery nor in the CSF validation cohort.

\section{Discussion}

In this study, we investigated the association of fatigue with clinical, brain MRI and CSF parameters in early MS.
Prevalence of fatigue in our cohort was in line with previous results in early MS patients from German MS Registry. ${ }^{29}$ Although fatigue can be observed in the absence of any other MS symptom, ${ }^{3}$ it increases with disability ${ }^{30-33}$ and disease duration, ${ }^{2,29,34}$ which was confirmed by our data. Furthermore, we found a robust association of fatigue and BDI, which is the recommended screening tool for depression in MS patients..$^{35}$ However, several BDI items overlap with fatigue symptoms (loss of energy, fatigue or difficulties in concentration) or are commonly observed in MS patients (sleep disorders, changes in appetite and sexual interest, irritability and emotional lability) $)^{36,37}$ whether as symptom of the disease or adverse drug reaction and it might be impossible to entirely disentangle fatigue and depression in MS patients. This in turn means that MS patients are likely to achieve BDI scores, indicating minimal/mild depression and excluding these patients from the analysis might introduce a bias in the results. This might not apply for MS patients with higher BDI scores, indicating 
clinically significant depression, which seems to resemble major depression..$^{38}$ Therefore, we repeated the analysis after exclusion of these patients without a meaningful change in the results.

We found weak correlations of fatigue with total and cortical grey matter that did not remain significant in the multivariate model that included EDSS score. We concluded that these correlations may not be specific for fatigue but rather reflect a longer or more severe disease course. Alternatively, the inclusion of an additional confounder might have decreased statistical power. In this case, however, one would expect consistent results among previous studies, ${ }^{5}$ which is not the case. To our knowledge, only few studies investigating the association of fatigue with MRI measures controlled for EDSS or disease duration. Damasceno and coworkers have described significant association of fatigue with lower volumes of caudate and accumbens nucleus in a cohort of 49 MS patients. ${ }^{33}$ Of note, $p$ values became moderate $(0.048,0.047)$ after correction for EDSS. We did not confirm these associations in our cohorts, which were both larger (68 and 233 patients). Yet we acknowledge that our patients had a shorter disease duration (mean disease duration $=4.3 / 5.0$ vs 6.2 years) and were less disabled (median EDSS = 1.0 and 1.5 vs 2.0).

Alternatively, fatigue in MS might be a consequence of chronic CNS inflammation. However, studies investigating different laboratory markers as potential biomarkers of fatigue in MS patients are inconclusive. $^{5}$ It is believed that inflammatory changes in the CNS parenchyma are reflected to a certain extent in the CSF compartment. Thus, we measured inflammatory parameters and immune cell subsets in the CSF compartment, but did not identify any significant association with the development of fatigue.

We acknowledge limitations of our study. A more detailed immune cell subtyping, for example, with memory and activation markers, might help to further investigate these contradictory findings. In our study, this was not possible as it was a post hoc analysis. With regard to MRI parameters, the investigation of diffuse WM changes and spinal cord pathology could add valuable information. Functional imaging may help to understand fatigue-related dysfunction at the network level, although changes identified through this technique are in principle compatible with either subtle structural damage or functional changes merely resulting from the inflammatory milieu. In a recent combined structural and functional MRI study, functional connectivity of basal ganglia correlated with fatigue severity in the absence of structural changes.
This supports the hypothesis of a rather functional than structural cause of fatigue, at least in early MS. ${ }^{39}$ Finally, we did not control for sleep disorders which might also contribute to fatigue in MS. 40

In summary, we found a robust relation of fatigue with disease severity. We could link fatigue neither to damage of certain brain regions nor to a certain type of inflammatory activity. This, however, does not prove that those relations do not exist. Rather, demonstration of such associations may necessitate more sophisticated laboratory or MRI methods. Still, coincidence with disease duration and severity must be accounted for. Gaining a deeper understanding of fatigue may be very challenging as different aspects of MS pathology, as well as the premorbid personality, may eventually determine the final common pathway of fatigue.

\section{Acknowledgements}

The authors thank Anne-Kristin Dahse, Verena Grummel, Regina Kohler, Nadine Miksch, Carina Nowak and Andreas Winkler for CSF analysis; Daniel Fröbel for assistance with management of the MRI database, Kirsten Brinkhoff, Sanja Kramberger and Kim Obergfell for the assessment of clinical and neuropsychological tests.

\section{Declaration of Conflicting Interests}

The author(s) declared the following potential conflicts of interest with respect to the research, authorship and/or publication of this article: V.B., P.S., R.C.S., V.P., B.K., T.K. and C.Z. had nothing to disclose; M.C.K. received travel fund from Merck Serono, Bayer HealthCare and Novartis, and received research support from Novartis and Medigene; D.B. received compensation for activities with Bayer HealthCare, Biogen Idec, Merck Serono and Novartis, and she is supported by the Abirisk Consortium; M.-M.H. received compensation for a lecture and travel grants from Bayer HealthCare and Biogen Idec; A.B. received grants from Bayer HealthCare, personal fees from Biogen, Merck Serono, Teva, Novartis and Genzyme, and compensations for clinical trials from Biogen, Novartis, Genzyme, Roche and Alexion Pharmaceuticals - outside the submitted work; J.S.K. received honoraria for scientific presentations from Philips Healthcare; B.H. served on scientific advisory boards for F. Hoffmann-La Roche Ltd., Novartis, Bayer Schering and Genentech, and received honoraria for lectures from Teva Pharmaceutical Industries, Biogen Idec, Medimmune and F. Hoffmann-La Roche Ltd. He received research support from Chugai Pharmaceuticals and F. Hoffmann-La Roche Ltd. He has filed a patent for the detection of antibodies and $\mathrm{T}$ 
cells against KIR4.1 in a subpopulation of MS patients and genetic determinants of neutralizing antibodies to interferon-beta; and M.M. received research support from Merck Serono and Novartis.

\section{Funding}

The author(s) disclosed receipt of the following financial support for the research, authorship and/or publication of this article: This work was supported by the Kommission für Klinische Forschung, Medical Faculty of the Technical University of Munich and the German competence network multiple sclerosis (KKNMS, grant no. 01GI1307B). B.H. received funding from the Deutsche Forschungsgemeinschaft DFG (TR 128).

\section{References}

1. Runia TF, Jafari N, Siepman DA, et al. Fatigue at time of CIS is an independent predictor of a subsequent diagnosis of multiple sclerosis. J Neurol Neurosurg Psychiatry 2015; 86: 543-546.

2. Kister I, Bacon TE, Chamot E, et al. Natural history of multiple sclerosis symptoms. Int J MS Care 2013; 15: 146-158.

3. Lebrun C, Cohen M and Clavelou P. Evaluation of quality of life and fatigue in radiologically isolated syndrome. Rev Neurol 2016; 172: 392-395.

4. Cavallari M, Palotai M, Glanz BI, et al. Fatigue predicts disease worsening in relapsing-remitting multiple sclerosis patients. Mult Scler 2016; 22: 1841-1849.

5. Patejdl R, Penner IK, Noack TK, et al. Multiple sclerosis and fatigue: A review on the contribution of inflammation and immune-mediated neurodegeneration. Autoimmun Rev 2016; 15: 210-220.

6. Bol Y, Duits AA, Lousberg R, et al. Fatigue and physical disability in patients with multiple sclerosis: A structural equation modeling approach. $J$ Behav Med 2010; 33: 355-363.

7. Morris G, Berk M, Galecki P, et al. The emerging role of autoimmunity in myalgic encephalomyelitis/ chronic fatigue syndrome (ME/cfs). Mol Neurobiol 2014; 49: 741-756.

8. Bradley AS, Ford B and Bansal AS. Altered functional B cell subset populations in patients with chronic fatigue syndrome compared to healthy controls. Clin Exp Immunol 2013; 172: 73-80.

9. Maes M, Bosmans E and Kubera M. Increased expression of activation antigens on $\mathrm{CD} 8+\mathrm{T}$ lymphocytes in Myalgic Encephalomyelitis/chronic fatigue syndrome: Inverse associations with lowered
CD19+ expression and CD4+/CD8+ ratio, but no associations with (auto)immune, leaky gut, oxidative and nitrosative stress biomarkers. Neuro Endocrinol Lett 2015; 36: 439-446.

10. Brenu EW, Broadley S, Nguyen T, et al. A preliminary comparative assessment of the role of CD8+ T Cells in chronic fatigue syndrome/myalgic encephalomyelitis and multiple sclerosis. J Immunol Res 2016; 2016: 9064529.

11. Fluge $\mathrm{O}$, Bruland $\mathrm{O}$, Risa $\mathrm{K}$, et al. Benefit from B-lymphocyte depletion using the anti-CD20 antibody rituximab in chronic fatigue syndrome. A double-blind and placebo-controlled study. PLOS ONE 2011; 6: e26358.

12. Dass S, Bowman SJ, Vital EM, et al. Reduction of fatigue in Sjogren syndrome with rituximab: Results of a randomised, double-blind, placebo-controlled pilot study. Ann Rheum Dis 2008; 67: 1541-1544.

13. De Flon P, Laurell K, Soderstrom L, et al. Improved treatment satisfaction after switching therapy to rituximab in relapsing-remitting MS. Mult Scler; 23: $1249-1257$.

14. Polman $\mathrm{CH}$, Reingold SC, Banwell B, et al. Diagnostic criteria for multiple sclerosis: 2010 revisions to the McDonald criteria. Ann Neurol 2011; 69: 292-302.

15. Penner IK, Raselli C, Stocklin M, et al. The Fatigue Scale for Motor and Cognitive Functions (FSMC): Validation of a new instrument to assess multiple sclerosis-related fatigue. Mult Scler 2009; 15: 1509-1517.

16. Beck AT, Steer RA and Brown GK. Manual for the Beck Depression Inventory-II. San Antonio, TX: Psychological Corporation, 1996.

17. Marrie RA, Cohen J, Stuve O, et al. A systematic review of the incidence and prevalence of comorbidity in multiple sclerosis: Overview. Mult Scler 2015; 21: 263-281.

18. Cepok S, Rosche B, Grummel V, et al. Short-lived plasma blasts are the main B cell effector subset during the course of multiple sclerosis. Brain 2005; 128: 1667-1676.

19. Shattuck DW, Mirza M, Adisetiyo V, et al. Construction of a 3D probabilistic atlas of human cortical structures. Neuroimage 2008; 39: 1064-1080.

20. Chard DT, Jackson JS, Miller DH, et al. Reducing the impact of white matter lesions on automated measures of brain gray and white matter volumes. J Magn Reson Imaging 2010; 32: 223-228.

21. Bezzola L, Merillat S, Gaser C, et al. Traininginduced neural plasticity in golf novices. J Neurosci 2011; 31: 12444-12448. 
22. Treadway MT, Waskom ML, Dillon DG, et al. Illness progression, recent stress, and morphometry of hippocampal subfields and medial prefrontal cortex in major depression. Biol Psychiatry 2015; 77: 285-294.

23. Muhlau M, Buck D, Forschler A, et al. White-matter lesions drive deep gray-matter atrophy in early multiple sclerosis: Support from structural MRI. Mult Scler 2013; 19: 1485-1492.

24. Keihaninejad S, Heckemann RA, Fagiolo G, et al. A robust method to estimate the intracranial volume across MRI field strengths (1.5T and 3T). Neuroimage 2010; 50: 1427-1437.

25. Good CD, Johnsrude IS, Ashburner J, et al. A voxelbased morphometric study of ageing in 465 normal adult human brains. Neuroimage 2001; 14: 21-36.

26. Geurts JJG, Calabrese M, Fisher E, et al. Measurement and clinical effect of grey matter pathology in multiple sclerosis. Lancet Neurol 2012; 11: 1082-1092.

27. Henry RG, Shieh M, Amirbekian B, et al. Connecting white matter injury and thalamic atrophy in clinically isolated syndromes. J Neurol Sci 2009; 282: 61-66.

28. R-Core-Team. $R$ : A language and environment for statistical computing, version 3.2.4. Vienna: $\mathrm{R}$ Foundation for Statistical Computing, 2016.

29. Stuke K, Flachenecker P, Zettl UK, et al. Symptomatology of MS: Results from the German MS Registry. J Neurol 2009; 256: 1932-1935.

30. Iriarte J, Subira ML and Castro P. Modalities of fatigue in multiple sclerosis: Correlation with clinical and biological factors. Mult Scler 2000; 6: 124-130.

31. Simpson S Jr., Tan H, Otahal P, et al. Anxiety, depression and fatigue at 5-year review following
CNS demyelination. Acta Neurol Scand 2016; 134: 403-413.

32. Calabrese M, Rinaldi F, Grossi $P$, et al. Basal ganglia and frontal/parietal cortical atrophy is associated with fatigue in relapsing-remitting multiple sclerosis. Mult Scler 2010; 16: 1220-1228.

33. Damasceno A, Damasceno BP and Cendes F. Atrophy of reward-related striatal structures in fatigued MS patients is independent of physical disability. Mult Scler 2016; 22: 822-829.

34. Tedeschi G, Dinacci D, Lavorgna L, et al. Correlation between fatigue and brain atrophy and lesion load in multiple sclerosis patients independent of disability. $J$ Neurol Sci 2007; 263: 15-19.

35. Goldman Consensus Group. The Goldman Consensus statement on depression in multiple sclerosis. Mult Scler 2005; 11: 328-337.

36. Figved N, Klevan G, Myhr KM, et al. Neuropsychiatric symptoms in patients with multiple sclerosis. Acta Psychiat Scand 2005; 112: 463-468.

37. Bamer AM, Johnson KL, Amtmann D, et al. Prevalence of sleep problems in individuals with multiple sclerosis. Mult Scler 2008; 14: 1127-1130.

38. Hasselmann H, Bellmann-Strobl J, Ricken R, et al. Characterizing the phenotype of multiple sclerosisassociated depression in comparison with idiopathic major depression. Mult Scler 2016; 22: 1476-1484.

39. Finke C, Schlichting J, Papazoglou S, et al. Altered basal ganglia functional connectivity in multiple sclerosis patients with fatigue. Mult Scler 2015; 21: 925-934.

40. Veauthier C and Paul F. Sleep disorders in multiple sclerosis and their relationship to fatigue. Sleep Med 2014; 15: 5-14.
Visit SAGE journals online journals.sagepub.com/ home/msj

( SAGE journals 